\title{
CONCENTRATION POINTS OF LEAST ENERGY SOLUTIONS TO THE BREZIS-NIRENBERG EQUATION WITH VARIABLE COEFFICIENTS
}

\author{
FUTOSHI TAKAHASHI \\ Department of Mathematics, Osaka City University \\ Sumiyoshi-ku, Osaka, 558-8585, Japan \\ E-mail: futoshi@sci.osaka-cu.ac.jp
}

1. Introduction. In this paper we consider the extensively studied problem

$$
\left(P_{\varepsilon, k}\right) \begin{cases}-\Delta u=u^{p}+\varepsilon k(x) u & \text { in } \Omega, \\ u>0 & \text { in } \Omega, \\ \left.u\right|_{\partial \Omega}=0 & \end{cases}
$$

where $\Omega$ is a smooth bounded domain in $\mathbf{R}^{N}, N \geq 4, p=(N+2) /(N-2)$ is the critical Sobolev exponent, $\varepsilon>0$ and $k \in C^{2}(\bar{\Omega})$ is a given function.

We are interested in the asymptotic behavior of blowing up solutions to $\left(P_{\varepsilon, k}\right)$ as $\varepsilon \rightarrow 0$.

Note that when $\Omega$ is star-shaped (with respect to 0 ), the Pohozaev identity yields the nonexistence of solutions to $\left(P_{\varepsilon, k}\right)$ if $k(x)+\frac{1}{2} x \cdot \nabla k(x) \leq 0$ for any $x \in \Omega$.

On the other hand, solutions to $\left(P_{\varepsilon, k}\right)$ on a general domain can be obtained by solving the constrained minimization problem

$$
S_{\varepsilon, k}=\inf _{\substack{u \in H_{0}^{1}(\Omega) \\\|u\|_{L^{p+1}(\Omega)}=1}}\left\{\int_{\Omega}|\nabla u|^{2} d x-\varepsilon \int_{\Omega} k(x) u^{2} d x\right\} .
$$

Let $S$ denote the best Sobolev constant. Let $\varepsilon>0$ be sufficiently small so that the operator $-\Delta-\varepsilon k(x)$ is coercive on $H_{0}^{1}(\Omega)$ (for example, it would be enough that $0<\varepsilon<\frac{(1-C) \lambda_{1}(\Omega)}{\|k\|_{L}(\Omega)}$ for some constant $0<C<1$, here $\lambda_{1}(\Omega)$ denotes the first eigenvalue of $-\Delta$ acting on $\left.H_{0}^{1}(\Omega)\right)$. Then, Brezis and Nirenberg proved that the conditions

(1) $k(x)>0$ somewhere on $\Omega$,

(2) $S_{\varepsilon, k}<S$,

2000 Mathematics Subject Classification: 35B40, 35J20.

The paper is in final form and no version of it will be published elsewhere. 
(3) $S_{\varepsilon, k}$ is achieved

are equivalent ([2], see also [1]).

From now on, we assume the condition (1), thus the existence of a minimizer $u_{\varepsilon}^{0}$ of $S_{\varepsilon, k}$ is assured. We may assume $u_{\varepsilon}^{0}>0$ by considering $\left|u_{\varepsilon}^{0}\right|$ if necessary. $S_{\varepsilon, k}$ is positive when $\varepsilon>0$ small, thus the Lagrange multiplier rule and elliptic regularity assure that

$$
\bar{u}_{\varepsilon}=\left(S_{\varepsilon, k}\right)^{\frac{N-2}{4}} u_{\varepsilon}^{0}
$$

is a smooth solution to $\left(P_{\varepsilon, k}\right)$. We call $\left(\bar{u}_{\varepsilon}\right)$ the least energy solutions to the problem $\left(P_{\varepsilon, k}\right)$.

In the following we consider only least energy solutions obtained by the method of Brezis and Nirenberg.

Note that the least energy solutions $\left(\bar{u}_{\varepsilon}\right)$ is also a minimizing sequence for the Sobolev best constant $S$, by the fact that $S_{\varepsilon, k}=S+o(1)$ as $\varepsilon \rightarrow 0$. Thus, by [3] Lemma I.1 and [7], it is known that $\left(\bar{u}_{\varepsilon}\right)$ concentrate at one point of $\bar{\Omega}$ : There exist $\lambda_{\varepsilon}>0$ with $\lambda_{\varepsilon} \rightarrow 0(\varepsilon \rightarrow 0)$ and $a_{\varepsilon} \in \Omega$ with $\lambda_{\varepsilon} / \operatorname{dist}\left(a_{\varepsilon}, \partial \Omega\right) \rightarrow 0(\varepsilon \rightarrow 0)$ such that, by choosing a subsequence if necessary, $a_{\varepsilon} \rightarrow a_{\infty} \in \bar{\Omega}$ and

$$
\begin{gathered}
\left\|\nabla\left(\bar{u}_{\varepsilon}-\alpha_{N} P U_{\lambda_{\varepsilon}, a_{\varepsilon}}\right)\right\|_{L^{2}(\Omega)} \rightarrow 0, \quad \text { where } \alpha_{N}=(N(N-2))^{\frac{N-2}{4}}, \\
\left|\nabla \bar{u}_{\varepsilon}\right|^{2} \stackrel{*}{\rightarrow} S^{\frac{N}{2}} \delta_{a_{\infty}}
\end{gathered}
$$

in the sense of Radon measures of $\bar{\Omega}$ as $\varepsilon \rightarrow 0$, where $\delta_{a_{\infty}}$ is a Dirac mass at $a_{\infty} \in \bar{\Omega}$. Here for $\lambda>0$ and $a \in \Omega, P U_{\lambda, a}(x)$ denotes the projection of $U_{\lambda, a}$ to $H_{0}^{1}(\Omega)$ defined by $P U_{\lambda, a}=U_{\lambda, a}-\varphi_{\lambda, a} \in H_{0}^{1}(\Omega)$ where $\varphi_{\lambda, a}$ is the harmonic extension of $\left.U_{\lambda, a}\right|_{\partial \Omega}$ to $\Omega$,

$$
U_{\lambda, a}(x)=\left(\frac{\lambda}{\lambda^{2}+|x-a|^{2}}\right)^{\frac{N-2}{2}}, \quad x \in \mathbf{R}^{N},
$$

is the unique (up to translation and dilation) positive solution of $-\Delta U=N(N-2) U^{p}$ in $\mathbf{R}^{N}$.

Concentration phenomena in elliptic problems involving critical Sobolev exponents like $\left(P_{\varepsilon, k}\right)$ are now widely studied. For the special case of $k \equiv 1$, see [4], [7], [8] and [9]. The Robin function of the domain plays an important role in these studies. Han and Rey showed that if $\left(u_{\varepsilon}\right)$ is a family of solutions of $\left(P_{\varepsilon, 1}\right)$ which concentrate at a point $a_{\infty} \in \bar{\Omega}$ in the sense of (1.4), then $a_{\infty}$ is interior of $\Omega$ and is a critical point of the (positive) Robin function

$$
R(a)=H(a, a), \quad a \in \Omega,
$$

where $H(x, a)$ is the regular part of the Green's function $G(x, a)$,

$$
H(x, a)=\frac{1}{(N-2) \omega_{N}}|x-a|^{2-N}-G(x, a),
$$

here $\omega_{N}$ is the $(N-1)$ dimensional volume of $S^{N-1}$. By the maximum principle and the Harnack inequality, we have

$$
C_{1} \leq R(a)(\operatorname{dist}(a, \partial \Omega))^{N-2} \leq C_{2}
$$


for some $C_{1}, C_{2}>0$ independent of $a \in \Omega$. Thus $R(a) \rightarrow \infty$ as $a \rightarrow \partial \Omega$. More precisely, we know

$$
R\left(a_{n}\right)=\frac{1}{(N-2) \omega_{N}}\left(\frac{1}{2 d_{n}}\right)^{N-2}+o\left(\frac{1}{d_{n}^{N-2}}\right)
$$

as $d_{n}=\operatorname{dist}\left(a_{n}, \partial \Omega\right) \rightarrow 0$ by $([8,(2.8)])$.

Later in [9], it is proved that any blow up point of least energy solutions is a minimum point of the Robin function on general bounded domains in $\mathbf{R}^{\mathbf{N}}, N \geq 4$.

Recently, Molle and Pistoia [6] studied a more general problem,

$$
\left(P_{\varepsilon, k}^{q}\right) \begin{cases}-\Delta u=u^{p}+\varepsilon k(x) u^{q} & \text { in } \Omega \\ u>0 & \text { in } \Omega, \\ \left.u\right|_{\partial \Omega}=0 & \end{cases}
$$

where $q \geq 1$ if $N \geq 5, q>1$ if $N=4$ and $q \neq p$.

They showed that if $\left(u_{\varepsilon}\right)$ is a family of solutions of $\left(P_{\varepsilon, k}^{q}\right)$ which concentrate at a point $a_{\infty} \in \Omega$ (interior point) in the sense of (1.3), then $a_{\infty}$ is a critical point of the function

$$
\psi_{q}(a)=k(a)\{R(a)\}^{\frac{q-p}{2}}, \quad a \in \Omega .
$$

In addition,

- $k\left(a_{\infty}\right)>0$ if $q<p$ (subcritical perturbation), and

- $k\left(a_{\infty}\right)<0$ if $q>p$ (supercritical perturbation).

Furthermore, they showed the existence of a family of solutions of $\left(P_{\varepsilon, k}^{q}\right)$ which concentrates at some point in $\Omega$ as $\varepsilon \rightarrow 0$. Especially, if $q<p$ and $\max _{\bar{\Omega}} k>0$, then there exists a family of solutions which concentrates at a maximum point of the function $\psi_{q}$.

Now, our main result in this note is the following

Theorem 1.1. Let $N \geq 4$. Assume that $\Omega_{+}:=\{a \in \Omega: k(a)>0\} \neq \phi$. Let $\left(\bar{u}_{\varepsilon}\right)$ be $a$ family of least energy solutions obtained by the method of Brezis-Nirenberg and $a_{\infty} \in \bar{\Omega}$ be a blow-up point of $\left(\bar{u}_{\varepsilon}\right)$ in the sense of (1.3). Then

(1) $a_{\infty} \in \Omega_{+}$, and

(2) $a_{\infty}$ maximizes the function $\psi_{1}(a)=k(a)\{R(a)\}^{\frac{-2}{N-2}}, a \in \Omega_{+}$:

$$
\psi_{1}\left(a_{\infty}\right)=\max _{a \in \Omega_{+}} \psi_{1}(a) .
$$

Mainly, our proof is almost the same as in [9] in which we treated the case when $k \equiv 1$, and the argument there originates from [5]. But there is also some improvement compared to the former calculations in [9].

2. Asymptotic behavior of $S_{\varepsilon, k}$. In this section, we will obtain an asymptotic formula of $S_{\varepsilon, k}$ as $\varepsilon \rightarrow 0$.

For a given sequence $\varepsilon_{n} \rightarrow 0$, let $u_{\varepsilon_{n}}^{0}$ be a positive minimizer for (1.1) and define

$$
v_{n}=S^{\frac{N-2}{4}} u_{\varepsilon_{n}}^{0}
$$

Then we see that $v_{n}$ and $\bar{u}_{\varepsilon_{n}}$ have the same concentration point $a_{\infty}$ and $\left|\nabla v_{n}\right|^{2} d x \stackrel{*}{\rightarrow}$ $S^{\frac{N}{2}} \delta_{a_{\infty}}$ in the sense of measures on $\bar{\Omega}$. 
Now, by a result of Rey ([8, Proposition 2]), we know there exists $\left(\alpha_{n}, \lambda_{n}, a_{n}\right) \in$ $\mathbf{R}_{+} \times \mathbf{R}_{+} \times \Omega$ such that

$$
v_{n}=\alpha_{n} P U_{\lambda_{n}, a_{n}}+w_{n}
$$

holds true for $n$ large, where

$$
\begin{aligned}
& \alpha_{n} \rightarrow \alpha_{N}=(N(N-2))^{\frac{N-2}{4}}, \\
& a_{n} \rightarrow a_{\infty}, \\
& \frac{\lambda_{n}}{d_{n}} \rightarrow 0 \quad \text { where } d_{n}=\operatorname{dist}\left(a_{n}, \partial \Omega\right), \\
& w_{n} \in E_{\lambda_{n}, a_{n}}, \\
& w_{n} \rightarrow 0 \text { in } H_{0}^{1}(\Omega)
\end{aligned}
$$

as $n \rightarrow \infty$. Here for $\lambda>0$ and $a \in \Omega$, we set $P U_{\lambda, a}=U_{\lambda, a}-\varphi_{\lambda, a} \in H_{0}^{1}(\Omega)$ where $\varphi_{\lambda, a}$ is the harmonic extension of $\left.U_{\lambda, a}\right|_{\partial \Omega}$ to $\Omega$, and

$$
\begin{aligned}
E_{\lambda, a}=\left\{w \in H_{0}^{1}(\Omega): 0\right. & =\int_{\Omega} \nabla w \cdot \nabla P U_{\lambda, a} d x \\
& =\int_{\Omega} \nabla w \cdot \nabla\left(\frac{\partial}{\partial a_{i}} P U_{\lambda, a}\right) d x \quad(i=1, \cdots, N) \\
& \left.=\int_{\Omega} \nabla w \cdot \nabla\left(\frac{\partial}{\partial \lambda} P U_{\lambda, a}\right) d x\right\} .
\end{aligned}
$$

Note that the estimate

$$
\left\|\varphi_{\lambda_{n}, a_{n}}\right\|_{L^{\infty}(\Omega)}=O\left(\frac{\lambda_{n}^{\frac{N-2}{2}}}{d_{n}^{N-2}}\right)
$$

holds by the maximum principle for harmonic functions and

$$
\varphi_{\lambda_{n}, a_{n}}\left(a_{n}\right)=(N-2) \omega_{N} \lambda_{n}^{\frac{N-2}{2}} R\left(a_{n}\right)+O\left(\frac{\lambda_{n}^{\frac{N+2}{2}}}{d_{n}^{N}}\right)
$$

by $[8$, Proposition 1$]$.

Let

$$
J_{n, k}=\int_{\Omega}\left|\nabla v_{n}\right|^{2} d x-\varepsilon_{n} \int_{\Omega} k(x) v_{n}^{2} d x .
$$

Then $S_{\varepsilon_{n}, k}=S^{1-\frac{N}{2}} J_{n, k}$, so in the following we calculate $J_{n, k}$ by using the expression (2.1).

The first lemma concerns the $H_{0}^{1}$ norm of the main part and is well known, see [9].

Lemma 2.1. Let $N \geq 4$. We have

$$
\int_{\Omega}\left|\nabla P U_{\lambda_{n}, a_{n}}\right|^{2} d x=N(N-2) A-(N-2)^{2} \omega_{N}^{2} R\left(a_{n}\right) \lambda_{n}^{N-2}+O\left(\frac{\lambda_{n}^{N}}{d_{n}^{N}}\left|\log \left(\frac{\lambda_{n}}{d_{n}}\right)\right|\right)
$$

as $n \rightarrow \infty$, where

$$
A=\int_{\mathbf{R}^{N}} U_{1,0}^{p+1} d x=\frac{\Gamma(N / 2)}{\Gamma(N)} \pi^{N / 2}
$$

Next we will prove 
LEMMA 2.2 (Asymptotic behavior of $L^{2}$ norm of the main part). When $N \geq 5$, we have

$$
\int_{\Omega} k(x) P U_{\lambda_{n}, a_{n}}^{2} d x=k\left(a_{n}\right) \omega_{N} C_{N} \lambda_{n}^{2}+o\left(\lambda_{n}^{2}\right) \quad \text { as } n \rightarrow \infty,
$$

where

$$
C_{N}=\int_{0}^{\infty} \frac{s^{N-1}}{\left(1+s^{2}\right)^{N-2}} d s=\frac{\Gamma\left(\frac{N}{2}\right) \Gamma\left(\frac{N-4}{2}\right)}{2 \Gamma(N-2)} .
$$

When $N=4$, we have

$$
\int_{\Omega} k(x) P U_{\lambda_{n}, a_{n}}^{2} d x=k\left(a_{n}\right) \omega_{4} \lambda_{n}^{2}\left|\log \lambda_{n}\right|+O\left(\frac{\lambda_{n}^{2}}{d_{n}}\left|\log \lambda_{n}\right|^{1 / 2}\right)+O\left(\frac{\lambda_{n}^{2}}{d_{n}^{2}}\right)
$$

as $n \rightarrow \infty$.

Proof. We extend $P U_{\lambda_{n}, a_{n}}$ and $\varphi_{\lambda_{n}, a_{n}}$ to $\mathbf{R}^{N}$ by setting $P U_{\lambda_{n}, a_{n}}=0$ and $\varphi_{\lambda_{n}, a_{n}}=$ $U_{\lambda_{n}, a_{n}}$, respectively, in $\mathbf{R}^{N} \backslash \Omega$.

First we treat the case $N \geq 5$. We have

$$
\begin{aligned}
\int_{\Omega} k(x) P U_{\lambda_{n}, a_{n}}^{2} d x & =\int_{\Omega} k(x) U_{\lambda_{n}, a_{n}}^{2} d x+\int_{\Omega} k(x) \varphi_{\lambda_{n}, a_{n}}^{2} d x \\
& +O\left(\left(\int_{\Omega} U_{\lambda_{n}, a_{n}}^{2} d x\right)^{1 / 2}\left(\int_{\Omega} \varphi_{\lambda_{n}, a_{n}}^{2} d x\right)^{1 / 2}\right) .
\end{aligned}
$$

We easily see

$$
\int_{\Omega} U_{\lambda_{n}, a_{n}}^{2} d x=O\left(\int_{\mathbf{R}^{N}} U_{\lambda_{n}, a_{n}}^{2} d x\right)=O\left(\lambda_{n}^{2}\right)
$$

When $N \geq 5$, we can check that

$$
\int_{\Omega} \varphi_{\lambda_{n}, a_{n}}^{2} d x=O\left(\frac{\lambda_{n}^{N-2}}{d_{n}^{N-4}}\right)
$$

Indeed, we represent the integral as

$$
\int_{\Omega} \varphi_{\lambda_{n}, a_{n}}^{2} d x=\int_{B_{d_{n}}\left(a_{n}\right)} \varphi_{\lambda_{n}, a_{n}}^{2} d x+\int_{\Omega \backslash B_{d_{n}}\left(a_{n}\right)} \varphi_{\lambda_{n}, a_{n}}^{2} d x .
$$

Now,

$$
\begin{aligned}
\int_{B_{d_{n}}\left(a_{n}\right)} \varphi_{\lambda_{n}, a_{n}}^{2} d x & =O\left(\left\|\varphi_{\lambda_{n}, a_{n}}\right\|_{L^{\infty}(\Omega)}^{2} \cdot \operatorname{vol}\left(B_{d_{n}}\left(a_{n}\right)\right)\right) \\
& =O\left(\left(\frac{\lambda_{n}^{N-2}}{d_{n}^{N-2}}\right)^{2} \cdot d_{n}^{N}\right)=O\left(\frac{\lambda_{n}^{N-2}}{d_{n}^{N-4}}\right)
\end{aligned}
$$

by $(2.2)$, and

$$
\begin{aligned}
\int_{\Omega \backslash B_{d_{n}}\left(a_{n}\right)} \varphi_{\lambda_{n}, a_{n}}^{2} d x & =O\left(\int_{\mathbf{R}^{N} \backslash B_{d_{n}}\left(a_{n}\right)} U_{\lambda_{n}, a_{n}}^{2} d x\right) \\
& =O\left(\int_{d_{n}}^{\infty}\left(\frac{\lambda_{n}}{\lambda_{n}^{2}+r^{2}}\right)^{N-2} r^{N-1} d r\right)=O\left(\frac{\lambda_{n}^{N-2}}{d_{n}^{N-4}}\right),
\end{aligned}
$$

since $0<\varphi_{\lambda_{n}, a_{n}}<U_{\lambda_{n}, a_{n}}$ in $\Omega$ and $\varphi_{\lambda_{n}, a_{n}}=U_{\lambda_{n}, a_{n}}$ on $\mathbf{R}^{N} \backslash \Omega$. Thus we obtain (2.7). 
By (2.6) and (2.7), we have

$$
\int_{\Omega} k(x) \varphi_{\lambda_{n}, a_{n}}^{2} d x+O\left(\left(\int_{\Omega} U_{\lambda_{n}, a_{n}}^{2} d x\right)^{1 / 2}\left(\int_{\Omega} \varphi_{\lambda_{n}, a_{n}}^{2} d x\right)^{1 / 2}\right)=o\left(\lambda_{n}^{2}\right) .
$$

Now, we estimate the term $\int_{\Omega} k(x) U_{\lambda_{n}, a_{n}}^{2} d x$. We split the integral as

$$
\int_{\Omega} k(x) U_{\lambda_{n}, a_{n}}^{2} d x=\int_{B_{d_{n}}\left(a_{n}\right)} k(x) U_{\lambda_{n}, a_{n}}^{2} d x+\int_{\Omega \backslash B_{d_{n}}\left(a_{n}\right)} k(x) U_{\lambda_{n}, a_{n}}^{2} d x .
$$

Making a Taylor expansion of $k(x)$ on $B_{d_{n}}\left(a_{n}\right)$, we have

$$
\begin{aligned}
\int_{B_{d_{n}}\left(a_{n}\right)} k(x) U_{\lambda_{n}, a_{n}}^{2} d x & =k\left(a_{n}\right) \int_{B_{d_{n}}\left(a_{n}\right)} U_{\lambda_{n}, a_{n}}^{2} d x+\nabla k\left(a_{n}\right) \cdot \int_{B_{d_{n}}\left(a_{n}\right)} U_{\lambda_{n}, a_{n}}^{2}\left(x-a_{n}\right) d x \\
& +\int_{B_{d_{n}}\left(a_{n}\right)} U_{\lambda_{n}, a_{n}}^{2} O\left(\left\|\nabla^{2} k\right\|_{L^{\infty}\left(B_{d_{n}}\left(a_{n}\right)\right)}\left|x-a_{n}\right|^{2}\right) d x .
\end{aligned}
$$

A calculation shows

$$
\begin{aligned}
\int_{B_{d_{n}}\left(a_{n}\right)} U_{\lambda_{n}, a_{n}}^{2} d x & =\omega_{N} \int_{0}^{d_{n}}\left(\frac{\lambda_{n}}{\lambda_{n}^{2}+r^{2}}\right)^{N-2} r^{N-1} d r \\
& =\omega_{N} \lambda_{n}^{2} \int_{0}^{d_{n} / \lambda_{n}} \frac{s^{N-1}}{\left(1+s^{2}\right)^{N-2}} d s=\omega_{N} \lambda_{n}^{2}\left(\int_{0}^{\infty}-\int_{d_{n} / \lambda_{n}}^{\infty}\right) \\
& =\omega_{N} \lambda_{n}^{2}\left(C_{N}+O\left(\left|\int_{d_{n} / \lambda_{n}}^{\infty} \frac{s^{N-1}}{\left(1+s^{2}\right)^{N-2}} d s\right|\right)\right) \\
& =\omega_{N} C_{N} \lambda_{n}^{2}+O\left(\frac{\lambda_{n}^{N-2}}{d_{n}^{N-4}}\right),
\end{aligned}
$$

here we have used the assumption $N \geq 5$. Since the integrand is odd, we also have

$$
\int_{B_{d_{n}}\left(a_{n}\right)} U_{\lambda_{n}, a_{n}}^{2}\left(x-a_{n}\right) d x=\overrightarrow{0} .
$$

Now, a direct calculation shows

$$
\begin{aligned}
& \int_{B_{d_{n}}\left(a_{n}\right)}\left|x-a_{n}\right|^{2} U_{\lambda_{n}, a_{n}}^{2} d x=\omega_{N} \int_{0}^{d_{n}}\left(\frac{\lambda_{n}}{\lambda_{n}^{2}+r^{2}}\right)^{N-2} r^{N+1} d r \\
= & \omega_{N} \lambda_{n}^{4} \int_{0}^{d_{n} / \lambda_{n}} \frac{s^{N+1}}{\left(1+s^{2}\right)^{N-2}} d s
\end{aligned}
$$

and

$$
\int_{0}^{d_{n} / \lambda_{n}} \frac{s^{N+1}}{\left(1+s^{2}\right)^{N-2}} d s= \begin{cases}O(1), & N>7 \\ O\left(\left|\log \left(\lambda_{n} / d_{n}\right)\right|\right), & N=6 \\ O\left(\lambda_{n}^{N-6} / d_{n}^{N-6}\right), & N<6\end{cases}
$$

Thus we obtain

$$
\int_{B_{d_{n}}\left(a_{n}\right)} k(x) U_{\lambda_{n}, a_{n}}^{2} d x=k\left(a_{n}\right) \omega_{N} C_{N} \lambda_{n}^{2}+o\left(\lambda_{n}^{2}\right)
$$


as $n \rightarrow \infty$. On the other hand, we estimate

$$
\begin{aligned}
\left|\int_{\Omega \backslash B_{d_{n}}\left(a_{n}\right)} k(x) U_{\lambda_{n}, a_{n}}^{2} d x\right|=O & \left(\|k\|_{L^{\infty}} \int_{\mathbf{R}^{N} \backslash B_{d_{n}}\left(a_{n}\right)} U_{\lambda_{n}, a_{n}}^{2} d x\right) \\
& =O\left(\int_{d_{n}}^{\infty}\left(\frac{\lambda_{n}}{\lambda_{n}^{2}+r^{2}}\right)^{N-2} r^{N-1} d r\right)=O\left(\frac{\lambda_{n}^{N-2}}{d_{n}^{N-4}}\right)
\end{aligned}
$$

as before when $N \geq 5$. Thus returning to (2.5), we have

$$
\int_{\Omega} k(x) P U_{\lambda_{n}, a_{n}}^{2} d x=k\left(a_{n}\right) \omega_{N} C_{N} \lambda_{n}^{2}+o\left(\lambda_{n}^{2}\right)
$$

as $n \rightarrow \infty$.

When $N=4$, we argue as follows.

We fix a bounded domain $\tilde{\Omega} \supset \supset$. Note that $\exists C_{1} \geq \tilde{d}_{n}:=\operatorname{dist}\left(a_{n}, \partial \tilde{\Omega}\right) \geq C_{2}>0$ for all $n$ since $a_{n} \in \Omega$. Denote $\tilde{R}=\operatorname{diam}(\tilde{\Omega})$. We extend $k \in C^{2}(\bar{\Omega})$ to $\tilde{\Omega}$ (which we also denote by $k$ ) so that $k \in C^{2}(\tilde{\Omega})$.

A calculation shows

$$
\begin{aligned}
\int_{B_{L}\left(a_{n}\right)} U_{\lambda_{n}, a_{n}}^{2} d x & =\omega_{4} \int_{r=0}^{r=L} \frac{\lambda_{n}^{2}}{\left(\lambda_{n}^{2}+r^{2}\right)^{2}} r^{3} d r=\omega_{4} \lambda_{n}^{2} \int_{s=0}^{s=L / \lambda_{n}} \frac{s^{3}}{\left(1+s^{2}\right)^{2}} d s \\
& =\omega_{4} \lambda_{n}^{2}\left[\frac{1}{2} \log \left(1+s^{2}\right)+\frac{1}{2}\left(1+s^{2}\right)^{-1}\right]_{s=0}^{s=L / \lambda_{n}} \\
& =\omega_{4} \lambda_{n}^{2}\left[\frac{1}{2} \log \left(\lambda_{n}^{2}+L^{2}\right)+\left|\log \lambda_{n}\right|+\frac{1}{2}\left(\frac{\lambda_{n}^{2}}{\lambda_{n}^{2}+L^{2}}\right)-\frac{1}{2}\right] \\
& =\omega_{4} \lambda_{n}^{2}\left|\log \lambda_{n}\right|+O\left(\lambda_{n}^{2}\right)
\end{aligned}
$$

for $n$ sufficiently large. Thus

$$
\int_{B_{\tilde{d}_{n}}\left(a_{n}\right)} U_{\lambda_{n}, a_{n}}^{2} d x=\omega_{4} \lambda_{n}^{2}\left|\log \lambda_{n}\right|+O\left(\lambda_{n}^{2}\right)
$$

since $\log \left(\lambda_{n}^{2}+\tilde{d}_{n}^{2}\right)=O(1)$ as $n \rightarrow \infty$ and

$$
\int_{\tilde{\Omega}} U_{\lambda_{n}, a_{n}}^{2} d x=\left(\int_{B_{\tilde{R}}\left(a_{n}\right)} U_{\lambda_{n}, a_{n}}^{2} d x\right)=O\left(\lambda_{n}^{2}\left|\log \lambda_{n}\right|\right) .
$$

Next, splitting the integral

$$
\int_{\tilde{\Omega}} \varphi_{\lambda_{n}, a_{n}}^{2} d x=\int_{B_{\tilde{d}_{n}}\left(a_{n}\right)} \varphi_{\lambda_{n}, a_{n}}^{2} d x+\int_{\tilde{\Omega} \backslash B_{\tilde{d}_{n}}\left(a_{n}\right)} \varphi_{\lambda_{n}, a_{n}}^{2} d x,
$$

and estimating

$$
\begin{aligned}
\int_{B_{\tilde{d}_{n}}\left(a_{n}\right)} \varphi_{\lambda_{n}, a_{n}}^{2} d x & =O\left(\left\|\varphi_{\lambda_{n}, a_{n}}\right\|_{L^{\infty}(\tilde{\Omega})} \cdot \int_{B_{\tilde{d}_{n}}\left(a_{n}\right)} \varphi_{\lambda_{n}, a_{n}} d x\right) \\
& =O\left(\left(\frac{\lambda_{n}}{d_{n}^{2}}\right) \int_{0}^{\tilde{d}_{n}} \frac{\lambda_{n}}{\lambda_{n}^{2}+r^{2}} r^{3} d r\right)=O\left(\frac{\lambda_{n}^{2}}{d_{n}^{2}}\right),
\end{aligned}
$$




$$
\begin{aligned}
\int_{\tilde{\Omega} \backslash B_{\tilde{d}_{n}}\left(a_{n}\right)} \varphi_{\lambda_{n}, a_{n}}^{2} d x & =O\left(\int_{B_{\tilde{R}}\left(a_{n}\right) \backslash B_{\tilde{d}_{n}}\left(a_{n}\right)} U_{\lambda_{n}, a_{n}}^{2} d x\right) \\
& =O\left(\int_{\tilde{d}_{n}}^{\tilde{R}}\left(\frac{\lambda_{n}}{\lambda_{n}^{2}+r^{2}}\right)^{2} r^{3} d r\right)=O\left(\lambda_{n}^{2}[\log r]_{r=\tilde{d}_{n}}^{r=\tilde{R}}\right)=O\left(\lambda_{n}^{2}\right),
\end{aligned}
$$

we have

$$
\int_{\tilde{\Omega}} \varphi_{\lambda_{n}, a_{n}}^{2} d x=O\left(\frac{\lambda_{n}^{2}}{d_{n}^{2}}\right) .
$$

Now, by (2.9) and (2.10), we have

$$
\begin{aligned}
\int_{\Omega} k(x) P U_{\lambda_{n}, a_{n}}^{2} d x= & \int_{\tilde{\Omega}} k(x) P U_{\lambda_{n}, a_{n}}^{2} d x \\
= & \int_{\tilde{\Omega}} k(x) U_{\lambda_{n}, a_{n}}^{2} d x+O\left(\left(\int_{\tilde{\Omega}} U_{\lambda_{n}, a_{n}}^{2} d x\right)^{1 / 2}\left(\int_{\tilde{\Omega}} \varphi_{\lambda_{n}, a_{n}}^{2} d x\right)^{1 / 2}\right) \\
& +\int_{\tilde{\Omega}} k(x) \varphi_{\lambda_{n}, a_{n}}^{2} d x \\
= & \int_{\tilde{\Omega}} k(x) U_{\lambda_{n}, a_{n}}^{2} d x+O\left(\frac{\lambda_{n}^{2}}{d_{n}^{2}}\right)+O\left(\frac{\lambda_{n}^{2}}{d_{n}}\left|\log \lambda_{n}\right|^{1 / 2}\right) .
\end{aligned}
$$

Finally, as before we split the integral

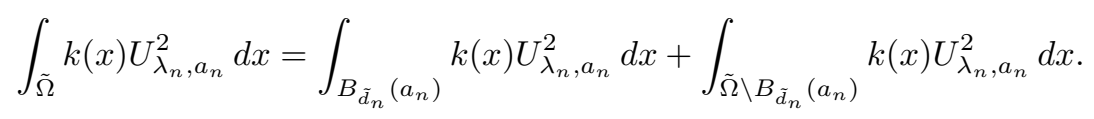

Taylor expansion of $k(x)$ leads to

$$
\begin{aligned}
\int_{B_{\tilde{d}_{n}}\left(a_{n}\right)} k(x) U_{\lambda_{n}, a_{n}}^{2} d x & =k\left(a_{n}\right) \int_{B_{\tilde{d}_{n}}\left(a_{n}\right)} U_{\lambda_{n}, a_{n}}^{2} d x+\nabla k\left(a_{n}\right) \cdot \int_{B_{\tilde{d}_{n}}\left(a_{n}\right)} U_{\lambda_{n}, a_{n}}^{2}\left(x-a_{n}\right) d x \\
& +O\left(\int_{B_{\tilde{d}_{n}}\left(a_{n}\right)}\left|x-a_{n}\right|^{2} U_{\lambda_{n}, a_{n}}^{2} d x\right) \\
& =k\left(a_{n}\right) \int_{B_{\tilde{d}_{n}}\left(a_{n}\right)} U_{\lambda_{n}, a_{n}}^{2} d x+0+O\left(\frac{\lambda_{n}^{2}}{d_{n}^{2}}\right) \\
& =k\left(a_{n}\right) \omega_{4} \lambda_{n}^{2}\left|\log \lambda_{n}\right|+O\left(\frac{\lambda_{n}^{2}}{d_{n}^{2}}\right),
\end{aligned}
$$

since

$$
\int_{B_{\tilde{d}_{n}}\left(a_{n}\right)}\left|x-a_{n}\right|^{2} U_{\lambda_{n}, a_{n}}^{2} d x=\omega_{4} \lambda_{n}^{4} \int_{0}^{\tilde{d}_{n} / \lambda_{n}} \frac{s^{5}}{\left(1+s^{2}\right)^{2}} d s=O\left(\lambda_{n}^{4} \cdot\left(\frac{\tilde{d}_{n}}{\lambda_{n}}\right)^{2}\right)
$$

and (2.8). On the other hand, we see that

$$
\int_{\tilde{\Omega} \backslash B_{\tilde{d}_{n}}\left(a_{n}\right)} k(x) U_{\lambda_{n}, a_{n}}^{2} d x=O\left(\int_{\tilde{d}_{n}}^{\tilde{R}}\left(\frac{\lambda_{n}}{\lambda_{n}^{2}+r^{2}}\right)^{2} r^{3} d r\right)=O\left(\lambda_{n}^{2}\right)=O\left(\frac{\lambda_{n}^{2}}{d_{n}^{2}}\right)
$$

since $C_{1} \geq \tilde{d}_{n} \geq C_{2}$. Going back to (2.11), we obtain Lemma 2.2 when $N=4$.

Since $w_{n} \in E_{\lambda_{n}, a_{n}}$ (see (2.2)), we have

$$
\int_{\Omega}\left|\nabla v_{n}\right|^{2} d x=\alpha_{n}^{2} \int_{\Omega}\left|\nabla P U_{\lambda_{n}, a_{n}}\right|^{2} d x+\int_{\Omega}\left|\nabla w_{n}\right|^{2} d x .
$$


Also we can estimate

$$
\int_{\Omega} k(x) v_{n}^{2} d x=\alpha_{n}^{2} \int_{\Omega} k(x) P U_{\lambda_{n}, a_{n}}^{2} d x+O\left(\left\|P U_{\lambda_{n}, a_{n}}\right\|_{L^{2}}\left\|w_{n}\right\|_{L^{2}}\right)+\int_{\Omega} k(x) w_{n}^{2} d x
$$

and

$$
\begin{aligned}
\varepsilon_{n} O\left(\left\|w_{n}\right\|_{L^{2}(\Omega)}^{2}\right) & =o\left(\left\|\nabla w_{n}\right\|_{L^{2}(\Omega)}^{2}\right) \\
O\left(\frac{\lambda_{n}^{N}}{d_{n}^{N}} \mid \log \left(\frac{\lambda_{n}}{d_{n}}\right)\right) & =o\left(\frac{\lambda_{n}^{N-2}}{d_{n}^{N-2}}\right), \\
\varepsilon_{n} O\left(\left\|P U_{\lambda_{n}, a_{n}}\right\|_{L^{2}}\left\|w_{n}\right\|_{L^{2}}\right) & =O\left(\varepsilon_{n}^{3 / 2}\left\|P U_{\lambda_{n}, a_{n}}\right\|_{L^{2}}^{2}\right)+O\left(\varepsilon_{n}^{1 / 2}\left\|\nabla w_{n}\right\|_{L^{2}}^{2}\right) \\
& =o\left(\varepsilon_{n}\left\|P U_{\lambda_{n}, a_{n}}\right\|_{L^{2}}^{2}\right)+o\left(\left\|\nabla w_{n}\right\|_{L^{2}}^{2}\right)
\end{aligned}
$$

by the Poincaré inequality. Combining these with Lemma 2.1 and Lemma 2.2, we have the following lemma concerning $J_{n, k}$ defined by (2.4):

LEMma 2.3 (Asymptotic behavior of $J_{n, k}$ ). We have

$$
\begin{aligned}
J_{n, k} & =\int_{\Omega}\left|\nabla v_{n}\right|^{2} d x-\varepsilon_{n} \int_{\Omega} k(x) v_{n}^{2} d x \\
& =\alpha_{n}^{2}\left\{N(N-2) A-(N-2)^{2} \omega_{N}^{2} R\left(a_{n}\right) \lambda_{n}^{N-2}-\varepsilon_{n} k\left(a_{n}\right) \omega_{N} C_{N} \lambda_{n}^{2}\right\} \\
& +\left\|\nabla w_{n}\right\|_{L^{2}(\Omega)}^{2} \\
& +o\left(\frac{\lambda_{n}^{N-2}}{d_{n}^{N-2}}\right)+o\left(\varepsilon_{n} \lambda_{n}^{2}\right) \quad \text { as } n \rightarrow \infty
\end{aligned}
$$

when $N \geq 5$, and

$$
\begin{aligned}
J_{n, k} & =\alpha_{n}^{2}\left\{8 A-4 \omega_{4}^{2} R\left(a_{n}\right) \lambda_{n}^{2}-\varepsilon_{n} k\left(a_{n}\right) \omega_{4} \lambda_{n}^{2}\left|\log \lambda_{n}\right|\right\} \\
& +\left\|\nabla w_{n}\right\|_{L^{2}(\Omega)}^{2} \\
& +o\left(\frac{\lambda_{n}^{2}}{d_{n}^{2}}\right)+o\left(\left\|\nabla w_{n}\right\|_{L^{2}(\Omega)}^{2}\right)+o\left(\varepsilon_{n} \lambda_{n}^{2}\left|\log \lambda_{n}\right|\right) \quad \text { as } n \rightarrow \infty
\end{aligned}
$$

when $N=4$.

To proceed further, we need to know the precise asymptotic behavior of $\alpha_{n}$ as $n \rightarrow \infty$. This is the subject of the next lemma.

Lemma 2.4 (Asymptotic behavior of $\alpha_{n}$ ). As $n \rightarrow \infty$, we have

$$
\begin{aligned}
\alpha_{n}^{2}=\alpha_{N}^{2}+2 \alpha_{N}^{2}\left(\frac{N-2}{N}\right)\left(\frac{\omega_{N}^{2}}{A}\right) R\left(a_{n}\right) \lambda_{n}^{N-2} & -\frac{N+2}{A(N-2)} \int_{\mathbf{R}^{N}} U_{\lambda_{n}, a_{n}}^{p-1} w_{n}^{2} d x \\
& +o\left(\left\|\nabla w_{n}\right\|_{L^{2}(\Omega)}^{2}\right)+o\left(\frac{\lambda_{n}^{N-2}}{d_{n}^{N-2}}\right)
\end{aligned}
$$

for $N \geq 4$, where $\alpha_{N}=(N(N-2))^{\frac{N-2}{4}}$.

Proof. After extending $v_{n}, P U_{\lambda_{n}, a_{n}}$, and $w_{n}$ by 0 outside $\Omega$, we have

$$
S^{N / 2}=\int_{\Omega} v_{n}^{p+1} d x=\int_{\mathbf{R}^{N}}\left|\alpha_{n} P U_{\lambda_{n}, a_{n}}+w_{n}\right|^{p+1} d x .
$$

We set $W_{n}:=-\alpha_{n} \varphi_{\lambda_{n}, a_{n}}+w_{n}$, here we extend $\varphi_{\lambda_{n}, a_{n}}$ to $\mathbf{R}^{N}$ as $U_{\lambda_{n}, a_{n}}$ on $\mathbf{R}^{N} \backslash \Omega$. 
By expanding the right hand side of (2.12), we have

$$
\begin{aligned}
S^{N / 2} & =\int_{\mathbf{R}^{N}}\left(\alpha_{n} U_{\lambda_{n}, a_{n}}+W_{n}\right)^{p+1} d x \\
& =\alpha_{n}^{p+1} \int_{\mathbf{R}^{N}} U_{\lambda_{n}, a_{n}}^{p+1} d x+(p+1) \alpha_{n}^{p} \int_{\mathbf{R}^{N}} U_{\lambda_{n}, a_{n}}^{p} W_{n} d x \\
& +\frac{(p+1) p}{2} \alpha_{n}^{p-1} \int_{\mathbf{R}^{N}} U_{\lambda_{n}, a_{n}}^{p-1} W_{n}^{2} d x \\
& +\left\{\begin{array}{l}
O\left(\int_{\mathbf{R}^{N}}\left|W_{n}\right|^{p+1} d x\right) \\
O\left(\int_{\mathbf{R}^{N}} U_{\lambda_{n}, a_{n}}^{p-2}\left|W_{n}\right|^{3} d x+\int_{\mathbf{R}^{N}}\left|W_{n}\right|^{p+1} d x\right)
\end{array} \quad(N=4,5) .\right.
\end{aligned}
$$

First, we know

$$
\alpha_{n}^{p+1} \int_{\mathbf{R}^{N}} U_{\lambda_{n}, a_{n}}^{p+1} d x=\alpha_{n}^{p+1} A .
$$

Next, by using the equation $-\Delta U_{\lambda_{n}, a_{n}}=N(N-2) U_{\lambda_{n}, a_{n}}^{p}$ in $\mathbf{R}^{N}$, we calculate

$$
\begin{aligned}
& (p+1) \alpha_{n}^{p} \int_{\mathbf{R}^{N}} U_{\lambda_{n}, a_{n}}^{p} W_{n} d x=\frac{2 \alpha_{n}^{p}}{(N-2)^{2}} \int_{\mathbf{R}^{N}}\left(-\Delta U_{\lambda_{n}, a_{n}}\right) W_{n} d x \\
= & \frac{2 \alpha_{n}^{p}}{(N-2)^{2}} \int_{\mathbf{R}^{N}} \nabla U_{\lambda_{n}, a_{n}} \cdot \nabla W_{n} d x \\
= & \frac{2 \alpha_{n}^{p}}{(N-2)^{2}} \int_{\mathbf{R}^{N}}\left(\nabla P U_{\lambda_{n}, a_{n}}+\nabla \varphi_{\lambda_{n}, a_{n}}\right) \cdot\left(-\alpha_{n} \nabla \varphi_{\lambda_{n}, a_{n}}+\nabla w_{n}\right) d x \\
= & \frac{-2 \alpha_{n}^{p+1}}{(N-2)^{2}} \int_{\mathbf{R}^{N}}\left|\nabla \varphi_{\lambda_{n}, a_{n}}\right|^{2} d x \\
= & -2 \alpha_{n}^{p+1} \omega_{N}^{2} R\left(a_{n}\right) \lambda_{n}^{N-2}+O\left(\frac{\lambda_{n}^{N}}{d_{n}^{N}}\left|\log \left(\frac{\lambda_{n}}{d_{n}}\right)\right|\right) .
\end{aligned}
$$

Here we have used the fact that $\varphi_{\lambda_{n}, a_{n}}$ is a harmonic function on $\Omega, w_{n} \in E_{\lambda_{n}, a_{n}}$ and

$$
\begin{aligned}
& \int_{\mathbf{R}^{N}}\left|\nabla \varphi_{\lambda_{n}, a_{n}}\right|^{2} d x=\int_{\mathbf{R}^{N}}\left|\nabla U_{\lambda_{n}, a_{n}}\right|^{2} d x-\int_{\mathbf{R}^{N}}\left|\nabla P U_{\lambda_{n}, a_{n}}\right|^{2} d x \\
= & (N-2)^{2} \omega_{N}^{2} R\left(a_{n}\right) \lambda_{n}^{N-2}+O\left(\frac{\lambda_{n}^{N}}{d_{n}^{N}}\left|\log \left(\frac{\lambda_{n}}{d_{n}}\right)\right|\right)
\end{aligned}
$$

by Lemma 2.1.

The third term of (2.13) is calculated as follows: we split

$$
\int_{\mathbf{R}^{N}} U_{\lambda_{n}, a_{n}}^{p-1} W_{n}^{2} d x=\int_{\mathbf{R}^{N} \backslash \Omega} U_{\lambda_{n}, a_{n}}^{p-1} W_{n}^{2} d x+\int_{\Omega} U_{\lambda_{n}, a_{n}}^{p-1} W_{n}^{2} d x:=I_{1}+I_{2} .
$$

Since $W_{n}=-\alpha_{n} U_{\lambda_{n}, a_{n}}$ on $\mathbf{R}^{N} \backslash \Omega$, the first term is estimated as

$$
I_{1}=\int_{\mathbf{R}^{N} \backslash \Omega} U_{\lambda_{n}, a_{n}}^{p-1} W_{n}^{2} d x=\alpha_{n}^{2} \int_{\mathbf{R}^{N} \backslash \Omega} U_{\lambda_{n}, a_{n}}^{p+1} d x=O\left(\int_{\mathbf{R}^{N} \backslash B_{d_{n}}\left(a_{n}\right)} U_{\lambda_{n}, a_{n}}^{p+1} d x\right) .
$$

Now we compute

$$
\int_{\mathbf{R}^{N} \backslash B_{d_{n}}\left(a_{n}\right)} U_{\lambda_{n}, a_{n}}^{p+1} d x=\omega_{N} \int_{d_{n}}^{\infty}\left(\frac{\lambda_{n}}{\lambda_{n}^{2}+r^{2}}\right)^{N} r^{N-1} d r=O\left(\frac{\lambda_{n}^{N}}{d_{n}^{N}}\right),
$$


so we have

$$
I_{1}=\int_{\mathbf{R}^{N} \backslash \Omega} U_{\lambda_{n}, a_{n}}^{p-1} W_{n}^{2} d x=O\left(\frac{\lambda_{n}^{N}}{d_{n}^{N}}\right) .
$$

Next, substituting $W_{n}$ by $-\alpha_{n} \varphi_{\lambda_{n}, a_{n}}+w_{n}$ in $I_{2}$, we have

$$
\begin{aligned}
I_{2} & =\int_{\Omega} U_{\lambda_{n}, a_{n}}^{p-1} W_{n}^{2} d x=\alpha_{n}^{2} \int_{\Omega} U_{\lambda_{n}, a_{n}}^{p-1} \varphi_{\lambda_{n}, a_{n}}^{2} d x+\int_{\mathbf{R}^{N}} U_{\lambda_{n}, a_{n}}^{p-1} w_{n}^{2} d x \\
& +O\left(\left(\int_{\Omega} U_{\lambda_{n}, a_{n}}^{p-1} w_{n}^{2} d x\right)^{1 / 2}\left(\int_{\Omega} U_{\lambda_{n}, a_{n}}^{p-1} \varphi_{\lambda_{n}, a_{n}}^{2} d x\right)^{1 / 2}\right) .
\end{aligned}
$$

The Hölder and the Sobolev inequalities imply

$$
\begin{aligned}
\int_{\Omega} U_{\lambda_{n}, a_{n}}^{p-1} w_{n}^{2} d x & =O\left(\left(\int_{\mathbf{R}^{N}} U_{\lambda_{n}, a_{n}}^{p+1} d x\right)^{\frac{p-1}{p+1}}\left(\int_{\Omega} w_{n}^{p+1} d x\right)^{\frac{2}{p+1}}\right) \\
& =O\left(\left\|\nabla w_{n}\right\|_{L^{2}(\Omega)}^{2}\right) .
\end{aligned}
$$

When we estimate the first term on the right hand side of (2.19), we consider the cases according to the dimension. First we assume $N \geq 5$. We split the integral

$$
\int_{\Omega} U_{\lambda_{n}, a_{n}}^{p-1} \varphi_{\lambda_{n}, a_{n}}^{2} d x=\int_{B_{d_{n}}\left(a_{n}\right)} U_{\lambda_{n}, a_{n}}^{p-1} \varphi_{\lambda_{n}, a_{n}}^{2} d x+\int_{\Omega \backslash B_{d_{n}}\left(a_{n}\right)} U_{\lambda_{n}, a_{n}}^{p-1} \varphi_{\lambda_{n}, a_{n}}^{2} d x .
$$

Then,

$$
\begin{aligned}
\int_{B_{d_{n}}\left(a_{n}\right)} U_{\lambda_{n}, a_{n}}^{p-1} \varphi_{\lambda_{n}, a_{n}}^{2} d x & =O\left(\left\|\varphi_{\lambda_{n}, a_{n}}\right\|_{L^{\infty}(\Omega)}^{2} \cdot \int_{B_{d_{n}}\left(a_{n}\right)} U_{\lambda_{n}, a_{n}}^{p-1} d x\right) \\
& =O\left(\left(\frac{\lambda_{n}^{N-2}}{d_{n}^{N-2}}\right)^{2} \cdot \lambda_{n}^{2} d_{n}^{N-4}\right)=O\left(\frac{\lambda_{n}^{N}}{d_{n}^{N}}\right)
\end{aligned}
$$

since

$$
\int_{B_{d_{n}}\left(a_{n}\right)} U_{\lambda_{n}, a_{n}}^{p-1} d x=\omega_{N} \int_{0}^{d_{n}}\left(\frac{\lambda_{n}}{\lambda_{n}^{2}+r^{2}}\right)^{2} r^{N-1} d r=O\left(\lambda_{n}^{2} d_{n}^{N-4}\right)
$$

for $N \geq 5$. On the other hand, by (2.17),

$$
\int_{\Omega \backslash B_{d_{n}}\left(a_{n}\right)} U_{\lambda_{n}, a_{n}}^{p-1} \varphi_{\lambda_{n}, a_{n}}^{2} d x=O\left(\int_{\mathbf{R}^{N} \backslash B_{d_{n}}\left(a_{n}\right)} U_{\lambda_{n}, a_{n}}^{p+1} d x\right)=O\left(\frac{\lambda_{n}^{N}}{d_{n}^{N}}\right) .
$$

Thus we have

$$
\int_{\Omega} U_{\lambda_{n}, a_{n}}^{p-1} \varphi_{\lambda_{n}, a_{n}}^{2} d x=O\left(\frac{\lambda_{n}^{N}}{d_{n}^{N}}\right)
$$

when $N \geq 5$.

When $N=4$, we have

$$
\begin{aligned}
\int_{B_{d_{n}}\left(a_{n}\right)} U_{\lambda_{n}, a_{n}}^{p-1} \varphi_{\lambda_{n}, a_{n}}^{2} d x & =O\left(\left\|\varphi_{\lambda_{n}, a_{n}}\right\|_{L^{\infty}(\Omega)}^{2} \cdot \int_{B_{d_{n}\left(a_{n}\right)}} U_{\lambda_{n}, a_{n}}^{p-1} d x\right) \\
& =O\left(\left(\frac{\lambda_{n}}{d_{n}^{2}}\right)^{2} \cdot \lambda_{n}^{2}\left(\log \left(1+\left(\frac{d_{n}}{\lambda_{n}}\right)^{2}\right)+O(1)\right)\right)=o\left(\frac{\lambda_{n}^{3}}{d_{n}^{3}}\right)
\end{aligned}
$$

since

$$
\int_{B_{d_{n}}\left(a_{n}\right)} U_{\lambda_{n}, a_{n}}^{p-1} d x=\omega_{4} \lambda_{n}^{2}\left[\frac{1}{2} \log \left(1+s^{2}\right)+\frac{1}{2}\left(1+s^{2}\right)^{-1}\right]_{s=0}^{s=\frac{d_{n}}{\lambda_{n}}}
$$


So arguing as above, we have

$$
\int_{\Omega} U_{\lambda_{n}, a_{n}}^{p-1} \varphi_{\lambda_{n}, a_{n}}^{2} d x=o\left(\frac{\lambda_{n}^{3}}{d_{n}^{3}}\right)
$$

when $N=4$. Combining (2.23) and (2.24), we conclude

$$
\int_{\Omega} U_{\lambda_{n}, a_{n}}^{p-1} \varphi_{\lambda_{n}, a_{n}}^{2} d x=o\left(\frac{\lambda_{n}^{N-1}}{d_{n}^{N-1}}\right)
$$

when $N \geq 4$.

Returning to (2.19) with (2.20) and (2.25), and using the Young inequality

$$
\left(\frac{\lambda_{n}}{d_{n}}\right)^{\frac{N-1}{2}}\|\nabla w\|_{L^{2}}=O\left(\left(\frac{\lambda_{n}}{d_{n}}\right)^{\frac{2 N-3}{2}}\right)+O\left(\left(\frac{\lambda_{n}}{d_{n}}\right)^{\frac{1}{2}}\|\nabla w\|_{L^{2}}^{2}\right),
$$

we have

$$
\int_{\mathbf{R}^{N}} U_{\lambda_{n}, a_{n}}^{p-1} W_{n}^{2} d x=\int_{\mathbf{R}^{N}} U_{\lambda_{n}, a_{n}}^{p-1} w_{n}^{2} d x+o\left(\frac{\lambda_{n}^{N-2}}{d_{n}^{N-2}}\right)+o\left(\|\nabla w\|_{L^{2}}^{2}\right) .
$$

Finally, by the Sobolev inequality and the inequality $(a+b)^{t} \leq C\left(a^{t}+b^{t}\right)$ for $C=2^{t-1}$ $(a, b>0, t>1)$, we have

$$
\begin{aligned}
\int_{\mathbf{R}^{N}}\left|W_{n}\right|^{p+1} d x & =O\left(\left(\int_{\mathbf{R}^{N}}\left|\nabla W_{n}\right|^{2} d x\right)^{\frac{p+1}{2}}\right) \\
& =O\left(\left(\int_{\mathbf{R}^{N}}\left|\nabla \varphi_{\lambda_{n}, a_{n}}\right|^{2} d x+\int_{\mathbf{R}^{N}}\left|\nabla w_{n}\right|^{2} d x\right)^{\frac{p+1}{2}}\right) \\
& =O\left(\left(\int_{\mathbf{R}^{N}}\left|\nabla \varphi_{\lambda_{n}, a_{n}}\right|^{2} d x\right)^{\frac{p+1}{2}}\right)+O\left(\left(\int_{\mathbf{R}^{N}}\left|\nabla w_{n}\right|^{2} d x\right)^{\frac{p+1}{2}}\right) .
\end{aligned}
$$

So by (2.16) and the estimate of the Robin function (1.5),

$$
\begin{aligned}
\int_{\mathbf{R}^{N}}\left|W_{n}\right|^{p+1} d x & =O\left(\left(\frac{\lambda_{n}^{N-2}}{d_{n}^{N-2}}\right)^{\frac{N}{N-2}}\right)+O\left(\left\|\nabla w_{n}\right\|_{L^{2}(\Omega)}^{\frac{2 N}{N-2}}\right) \\
& =O\left(\frac{\lambda_{n}^{N}}{d_{n}^{N}}\right)+o\left(\left\|\nabla w_{n}\right\|_{L^{2}(\Omega)}^{2}\right) .
\end{aligned}
$$

When $N=4,5$, we also need to estimate the term $\int_{\mathbf{R}^{N}} U_{\lambda_{n}, a_{n}}^{p-2} W_{n}^{3} d x$. But the calculation is almost the same. Indeed, by the Hölder inequality and (2.27), we have

$$
\begin{aligned}
\int_{\mathbf{R}^{N}} U_{\lambda_{n}, a_{n}}^{p-2}\left|W_{n}\right|^{3} d x & =O\left(\left(\int_{\mathbf{R}^{N}}\left|W_{n}\right|^{p+1} d x\right)^{\frac{3}{p+1}}\right) \times O\left(\left(\int_{\mathbf{R}^{N}} U_{\lambda_{n}, a_{n}}^{p+1} d x\right)^{\frac{p-2}{p+1}}\right) \\
& =\left(O\left(\frac{\lambda_{n}^{N}}{d_{n}^{N}}\right)+O\left(\left\|\nabla w_{n}\right\|_{L^{2}(\Omega)}^{p+1}\right)\right)^{\frac{3}{p+1}} \times O(1) \\
& =O\left(\left(\frac{\lambda_{n}^{N}}{d_{n}^{N}}\right)\right)^{\frac{3(N-2)}{2 N}}+O\left(\left\|\nabla w_{n}\right\|_{L^{2}(\Omega)}^{\frac{2 N}{N-2}}\right)^{\frac{3(N-2)}{2 N}} \\
& =o\left(\frac{\lambda_{n}^{N-2}}{d_{n}^{N-2}}\right)+o\left(\left\|\nabla w_{n}\right\|_{L^{2}(\Omega)}^{2}\right) .
\end{aligned}
$$

Here we have used the inequality $(a+b)^{t} \leq\left(a^{t}+b^{t}\right)$ for $a, b \geq$ and $0<t<1$. 
Inserting $(2.14),(2.15),(2.26),(2.27)$ (and $(2.28)$ when $N=4,5)$ to $(2.13)$, we obtain

$$
\begin{aligned}
S^{N / 2} & =\alpha_{n}^{p+1} A-2 \alpha_{n}^{p+1} \cdot \omega_{N}^{2} R\left(a_{n}\right) \lambda_{n}^{N-2} \\
& +\frac{(p+1) p}{2} \alpha_{n}^{p-1} \int_{\mathbf{R}^{N}} U_{\lambda_{n}, a_{n}}^{p-1} w_{n}^{2} d x+o\left(\frac{\lambda_{n}^{N-2}}{d_{n}^{N-2}}\right)+o\left(\|\nabla w\|_{L^{2}}^{2}\right) .
\end{aligned}
$$

Dividing both sides by $A$ and noting that $\frac{S^{N / 2}}{A}=\alpha_{N}^{p+1}$, we have

$$
\begin{gathered}
\alpha_{N}^{p+1}=\alpha_{n}^{p+1}-\alpha_{n}^{p+1}\left(\frac{2 \omega_{N}^{2}}{A}\right) R\left(a_{n}\right) \lambda_{n}^{N-2} \\
+\frac{p(p+1)}{2} \frac{\alpha_{n}^{p-1}}{A} \int_{\mathbf{R}^{N}} U_{\lambda_{n}, a_{n}}^{p-1} w_{n}^{2} d x+o\left(\frac{\lambda_{n}^{N-2}}{d_{n}^{N-2}}\right)+o\left(\|\nabla w\|_{L^{2}}^{2}\right) .
\end{gathered}
$$

Since $\alpha_{n}^{p+1}=\alpha_{N}^{p+1}+o(1)$ and $R\left(a_{n}\right)=O\left(\frac{1}{d_{n}^{N-2}}\right)$, we know

$$
\alpha_{n}^{p+1} R\left(a_{n}\right) \lambda_{n}^{N-2}=\alpha_{N}^{p+1} R\left(a_{n}\right) \lambda_{n}^{N-2}+o\left(\frac{\lambda_{n}^{N-2}}{d_{n}^{N-2}}\right) .
$$

Similarly, we have

$$
\alpha_{n}^{p-1} \int_{\mathbf{R}^{N}} U_{\lambda_{n}, a_{n}}^{p-1} w_{n}^{2} d x=\alpha_{N}^{p-1} \int_{\mathbf{R}^{N}} U_{\lambda_{n}, a_{n}}^{p-1} w_{n}^{2} d x+o\left(\|\nabla w\|_{L^{2}}^{2}\right) .
$$

Substituting these in $(2.29)$, we have

$$
\begin{aligned}
\alpha_{n}^{p+1} & =\alpha_{N}^{p+1}+\alpha_{N}^{p+1}\left(\frac{2 \omega_{N}^{2}}{A}\right) R\left(a_{n}\right) \lambda_{n}^{N-2} \\
& -\frac{p(p+1)}{2} \frac{\alpha_{N}^{p-1}}{A} \int_{\mathbf{R}^{N}} U_{\lambda_{n}, a_{n}}^{p-1} w_{n}^{2} d x+o\left(\frac{\lambda_{n}^{N-2}}{d_{n}^{N-2}}\right)+o\left(\|\nabla w\|_{L^{2}}^{2}\right),
\end{aligned}
$$

which implies

$$
\begin{aligned}
\alpha_{n}^{p+1} & =\alpha_{N}^{p+1}\left\{1+\left(\frac{2 \omega_{N}^{2}}{A}\right) R\left(a_{n}\right) \lambda_{n}^{N-2}-\frac{(p+1) p}{2 A \alpha_{N}^{2}} \int_{\mathbf{R}^{N}} U_{\lambda_{n}, a_{n}}^{p-1} w_{n}^{2} d x\right\} \\
& +o\left(\left\|\nabla w_{n}\right\|_{L^{2}(\Omega)}^{2}\right)+o\left(\frac{\lambda_{n}^{N-2}}{d_{n}^{N-2}}\right) .
\end{aligned}
$$

By Taylor expansion $(1+x)^{\frac{2}{p+1}}=1+\frac{2}{p+1} x+o(x)$ as $x \rightarrow 0$, we conclude that

$$
\begin{aligned}
\alpha_{n}^{2} & =\alpha_{N}^{2}\left\{1+\left(\frac{2}{p+1}\right)\left(\frac{2 \omega_{N}^{2}}{A}\right) R\left(a_{n}\right) \lambda_{n}^{N-2}-\frac{p}{A \alpha_{N}^{2}} \int_{\mathbf{R}^{N}} U_{\lambda_{n}, a_{n}}^{p-1} w_{n}^{2} d x\right\} \\
& +o\left(\left\|\nabla w_{n}\right\|_{L^{2}(\Omega)}^{2}\right)+o\left(\frac{\lambda_{n}^{N-2}}{d_{n}^{N-2}}\right)
\end{aligned}
$$

as $n \rightarrow \infty$.

This completes the proof of Lemma 2.4.

Combining Lemma 2.3 and Lemma 2.4, and noting $\alpha_{N}^{2} N(N-2) A=S^{N / 2}$, we obtain: 
Proposition 2.5 (Asymptotic behavior of $S_{\varepsilon_{n}, k}$ ). As $n \rightarrow \infty$, we have

$$
\begin{aligned}
S_{\varepsilon_{n}, k} & =\inf _{\substack{v \in H_{0}^{1}(\Omega) \\
\|v\|_{L^{p+1}(\Omega)}=1}}\left\{\int_{\Omega}|\nabla v|^{2} d x-\varepsilon_{n} \int_{\Omega} k(x) v^{2} d x\right\} \\
& =S \cdot S^{-\frac{N}{2}} J_{n, k}=S+S\left(\frac{N-2}{N}\right)\left(\frac{\omega_{N}^{2}}{A}\right) R\left(a_{n}\right) \lambda_{n}^{N-2} \\
& -\varepsilon_{n} k\left(a_{n}\right)\left(\frac{S \omega_{N} C_{N}}{N(N-2) A}\right) \lambda_{n}^{2} \\
& +S^{(2-N) / 2}\left\{\left\|\nabla w_{n}\right\|_{L^{2}}^{2}-N(N+2) \int_{\mathbf{R}^{N}} U_{\lambda_{n}, a_{n}}^{p-1} w_{n}^{2} d x\right\} \\
& +o\left(\frac{\lambda_{n}^{N-2}}{d_{n}^{N-2}}\right)+o\left(\left\|\nabla w_{n}\right\|_{L^{2}(\Omega)}^{2}\right)+o\left(\varepsilon_{n} \lambda_{n}^{2}\right)
\end{aligned}
$$

when $N \geq 5$, and

$$
\begin{aligned}
S_{\varepsilon_{n}, k} & =S+\frac{S}{2}\left(\frac{\omega_{4}^{2}}{A}\right) R\left(a_{n}\right) \lambda_{n}^{2}-\varepsilon_{n} k\left(a_{n}\right)\left(\frac{S \omega_{4}}{8 A}\right) \lambda_{n}^{2}\left|\log \lambda_{n}\right| \\
& +S^{-1}\left\{\left\|\nabla w_{n}\right\|_{L^{2}}^{2}-24 \int_{\mathbf{R}^{N}} U_{\lambda_{n}, a_{n}}^{2} w_{n}^{2} d x\right\} \\
& +o\left(\frac{\lambda_{n}^{2}}{d_{n}^{2}}\right)+o\left(\left\|\nabla w_{n}\right\|_{L^{2}(\Omega)}^{2}\right)+o\left(\varepsilon_{n} \lambda_{n}^{2}\left|\log \lambda_{n}\right|\right)
\end{aligned}
$$

when $N=4$.

To proceed further, we need the nondegeneracy result first shown by Rey ([8, Appendix D]).

Lemma 2.6 (Nondegeneracy inequality). There exists a constant $C>0$ which depends only on the dimension $N$ such that for any $w_{n} \in E_{\lambda_{n}, a_{n}}$,

$$
\int_{\mathbf{R}^{N}}\left|\nabla w_{n}\right|^{2} d x-N(N+2) \int_{\mathbf{R}^{N}} U_{\lambda_{n}, a_{n}}^{p-1} w_{n}^{2} d x \geq C \int_{\mathbf{R}^{N}}\left|\nabla w_{n}\right|^{2} d x .
$$

Furthermore, we need the appropriate bound of the value $S_{\varepsilon_{n}, k}$ from the above. The following Lemma is proved by the same argument of Lemma 2.7 in [9], so we omit the proof.

Lemma 2.7 (Upper bound of $S_{\varepsilon, k}$ ). For any $a \in \Omega_{+}=\{a \in \Omega: k(a)>0\}$ and $\rho>0$, there exists an $\varepsilon_{0}=\varepsilon_{0}(a, \rho)$ such that if $\varepsilon \in\left(0, \varepsilon_{0}\right)$, then

$$
S_{\varepsilon, k} \leq S-\left(\frac{N-4}{N-2}\right) \varepsilon k(a)\left\{\frac{S \omega_{N} C_{N}}{N(N-2) A}-\rho\right\}\left[\frac{2 C_{N} \varepsilon k(a)}{(N-2)^{3} \omega_{N} R(a)}\right]^{\frac{2}{N-4}}
$$

when $N \geq 5$, and

$$
S_{\varepsilon, k} \leq S-\frac{S \varepsilon k(a) \omega_{4}}{16 A e} \exp \left(-\frac{8 \omega_{4} R(a)+\varepsilon k(a) / e+2 \rho}{\varepsilon k(a)}\right)
$$

when $N=4$. 
3. Proof of Theorem. In this section, we prove Theorem 1.1.

The following elementary facts are important in the argument: for constants $C_{A}, C_{B}$ $>0$, the function

$$
f_{N}(\lambda)=S+C_{A} \lambda^{N-2}-C_{B} \lambda^{2}
$$

has the unique global minimum value

$$
\min _{\lambda>0} f_{N}(\lambda)=S-\left(\frac{N-4}{N-2}\right) C_{B}\left(\frac{2 C_{B}}{(N-2) C_{A}}\right)^{\frac{2}{N-4}}
$$

when $N \geq 5$, and

$$
f_{4}(\lambda)=S+C_{A} \lambda^{2}-C_{B} \lambda^{2}|\log \lambda|, \quad 0<\lambda<1,
$$

has the unique global minimum value

$$
\min _{\lambda>0} f_{4}(\lambda)=S-\left(\frac{C_{B}}{2 e}\right) \exp \left(-\frac{2 C_{A}}{C_{B}}\right)
$$

when $N=4$.

Now, we treat the case $N \geq 5$. Set

$$
K_{1}=S\left(\frac{N-2}{N}\right)\left(\frac{\omega_{N}^{2}}{A}\right), \quad K_{2}=\frac{S \omega_{N} C_{N}}{N(N-2) A} .
$$

First, we prove that $k\left(a_{n}\right)>0$ for $n$ sufficiently large. Assume the contrary that there exists a subsequence such that $k\left(a_{n}\right) \leq 0$. In addition if $k\left(a_{n}\right) \leq-C<0$ for some $C>0$ independent of $n$, then Proposition 2.5 and Lemma 2.6 yield the inequality $S_{\varepsilon_{n}, k} \geq S$. This is a contradiction to the fact $S>S_{\varepsilon_{n}, k}$ by Brezis and Nirenberg, see Introduction. Thus $k\left(a_{n}\right) \rightarrow 0$ for every sequence with $k\left(a_{n}\right) \leq 0$.

On the other hand, by the result of Brezis and Nirenberg, Proposition 2.5, Lemma 2.6 and (1.5), we have $C_{1}>0$ independent of $n$ such that

$$
S>S_{\varepsilon_{n}, k} \geq S+C_{1} \lambda_{n}^{N-2}-\left(k\left(a_{n}\right) K_{2}+p_{n}\right) \varepsilon_{n} \lambda_{n}^{2}
$$

for some $p_{n}>0, p_{n} \rightarrow 0$. Therefore we have

$$
C_{B}(n):=K_{2} k\left(a_{n}\right)+p_{n}>0
$$

for $n$ large. Thus by (3.1), we obtain

$$
S_{\varepsilon_{n}, k} \geq S-\left(\frac{N-4}{N-2}\right) C_{B}(n) \varepsilon_{n}\left(\frac{2 C_{B}(n) \varepsilon_{n}}{(N-2) C_{1}}\right)^{\frac{2}{N-4}} .
$$

Connecting this with the upper bound

$$
S_{\varepsilon_{n}, k} \leq S-C_{2} \varepsilon_{n}^{1+\frac{2}{N-4}}
$$

for some $C_{2}>0$, which is assured by Lemma 2.7, we have a contradiction since we have seen that $C_{B}(n) \rightarrow 0$ as $n \rightarrow 0$. Thus we have proved $k\left(a_{n}\right)>0$ for $n$ sufficiently large.

The same argument shows that when $k\left(a_{n}\right)>0$ for $n$ sufficiently large, it cannot happen that $k\left(a_{\infty}\right)=\lim _{n \rightarrow \infty} k\left(a_{n}\right)=0$.

Next, we will show that the blow up point $a_{\infty}$ is in the interior of $\Omega$. Suppose the contrary. Then $a_{\infty} \in \partial \Omega$ and $d_{n}=d\left(a_{n}, \partial \Omega\right) \rightarrow 0$ as $n \rightarrow \infty$. Then by Proposition 2.5, 
Lemma 2.6, (1.5) and the fact that $k\left(a_{n}\right) \geq C>0$ for large $n$, we can find constants $C_{1}, C_{2}, C_{3}>0$ such that when $N \geq 5$,

$$
\begin{aligned}
S_{\varepsilon_{n}, k} & =S+S\left(\frac{N-2}{N}\right)\left(\frac{\omega_{N}^{2}}{A}\right) R\left(a_{n}\right) \lambda_{n}^{N-2} \\
& -\varepsilon_{n} k\left(a_{n}\right)\left(\frac{S \omega_{N} C_{N}}{N(N-2) A}\right) \lambda_{n}^{2} \\
& +S^{(2-N) / 2}\left\{\left\|\nabla w_{n}\right\|_{L^{2}}^{2}-N(N+2) \int_{\mathbf{R}^{N}} U_{\lambda_{n}, a_{n}}^{p-1} w_{n}^{2} d x\right\} \\
& +o\left(\frac{\lambda_{n}^{N-2}}{d_{n}^{N-2}}\right)+o\left(\left\|\nabla w_{n}\right\|_{L^{2}(\Omega)}^{2}\right)+o\left(\varepsilon_{n} \lambda_{n}^{2}\right) \\
& \geq S+C_{1}\left(\frac{\lambda_{n}^{N-2}}{d_{n}^{N-2}}\right)-C_{2} \varepsilon_{n} \lambda_{n}^{2} \\
& \geq S-\left(\frac{N-4}{N-2}\right) C_{2} \varepsilon_{n}\left\{\frac{2 C_{2} \varepsilon_{n}}{(N-2) C_{1}\left(\frac{1}{d_{n}^{N-2}}\right)}\right\}^{\frac{2}{N-4}} \\
& =S-C_{3} \varepsilon_{n}^{\frac{N-2}{N-4}} d_{n}^{\frac{2(N-2)}{N-4}}=S+o\left(\varepsilon_{n}^{\frac{N-2}{N-4}}\right),
\end{aligned}
$$

since we assumed $d_{n} \rightarrow 0$. Here we used (3.1) in deriving the second inequality.

On the other hand, we know that $S_{\varepsilon_{n}, k} \leq S-C \varepsilon_{n}^{\frac{N-2}{N-4}}$ for some $C>0$ by Lemma 2.7. This contradicts the above estimate, so we conclude that $a_{\infty}$ is in the interior of $\Omega$.

Now, since we have proved that $d_{n} \geq C$ for some constant $C>0$ uniformly in $n$, we may drop $d_{n}$ in the asymptotic formula of Proposition 2.5. Then we can find $p_{n}, q_{n}>0$, $p_{n}, q_{n} \rightarrow 0$ such that

$$
\begin{aligned}
S_{\varepsilon_{n}, k} & =S+K_{1} R\left(a_{n}\right) \lambda_{n}^{N-2}-\varepsilon_{n} k\left(a_{n}\right) K_{2} \lambda_{n}^{2} \\
& +S^{(2-N) / 2}\left\{\left\|\nabla w_{n}\right\|_{L^{2}}^{2}-N(N+2) \int_{\mathbf{R}^{N}} U_{\lambda_{n}, a_{n}}^{p-1} w_{n}^{2} d x\right\} \\
& +o\left(\lambda_{n}^{N-2}\right)+o\left(\left\|\nabla w_{n}\right\|_{L^{2}(\Omega)}^{2}\right)+o\left(\varepsilon_{n} \lambda_{n}^{2}\right) \\
& \geq S+\left(K_{1} R\left(a_{n}\right)-p_{n}\right) \lambda_{n}^{N-2}-\left(K_{2} k\left(a_{n}\right)+q_{n}\right) \varepsilon_{n} \lambda_{n}^{2} \\
& \geq S-\left(\frac{N-4}{N-2}\right) \varepsilon_{n}\left(K_{2} k\left(a_{n}\right)+q_{n}\right)\left[\frac{2 \varepsilon_{n}\left(K_{2} k\left(a_{n}\right)+q_{n}\right)}{(N-2)\left(K_{1} R\left(a_{n}\right)-p_{n}\right)}\right]^{\frac{2}{N-4}} .
\end{aligned}
$$

The last inequality of (3.3) follows again by (3.1) and the fact that $K_{2} k\left(a_{n}\right)+q_{n}>0$ for $n$ large.

On the other hand, Lemma 2.7 gives an upper bound

$$
S_{\varepsilon_{n}, k} \leq S-\left(K_{2}-\rho\right) \varepsilon_{n} k(a)\left[\frac{2 K_{2} \varepsilon_{n} k(a)}{(N-2) K_{1} R(a)}\right]^{\frac{2}{N-4}}
$$

for any $a \in \Omega_{+}$and $\rho>0$ sufficiently small. Therefore by combining these, we have

$$
\begin{aligned}
& \left(K_{2} k\left(a_{n}\right)+q_{n}\right) \varepsilon_{n}\left[\frac{2 \varepsilon_{n}\left(K_{2} k\left(a_{n}\right)+q_{n}\right)}{(N-2)\left(K_{1} R\left(a_{n}\right)-p_{n}\right)}\right]^{\frac{2}{N-4}} \\
& \geq\left(K_{2}-\rho\right) k(a) \varepsilon_{n}\left[\frac{2 K_{2} \varepsilon_{n} k(a)}{(N-2) K_{1} R(a)}\right]^{\frac{2}{N-4}} .
\end{aligned}
$$


Dividing both sides by $\varepsilon_{n}^{\frac{N-2}{N-4}}$, letting $n \rightarrow \infty$ and $\rho \rightarrow 0$, we check that $a_{\infty}$ will maximize

$$
k(a)\left(\frac{k(a)}{R(a)}\right)^{\frac{2}{N-4}}=\left(\psi_{1}(a)\right)^{\frac{N-2}{N-4}} .
$$

When $N=4$, the fact that $k\left(a_{n}\right)>0$ for $n$ large and $k\left(a_{\infty}\right) \neq 0$ is proved similarly as in the proof when $N \geq 5$.

The proof of the fact that the blow up point belongs to the interior of $\Omega$ is also the same. We give a proof for the reader's convenience. Since $k\left(a_{n}\right) \geq C>0$ uniformly in $n$ large, we have some $C_{1}, C_{2}>0$ such that

$$
\begin{aligned}
S_{\varepsilon_{n}, k} & =S+\left(\frac{S \omega_{4}^{2}}{2 A}\right) R\left(a_{n}\right) \lambda_{n}^{2}-\varepsilon_{n} k\left(a_{n}\right)\left(\frac{S \omega_{4}}{8 A}\right) \lambda_{n}^{2}\left|\log \lambda_{n}\right| \\
& +S^{-1}\left\{\left\|\nabla w_{n}\right\|_{L^{2}}^{2}-24 \int_{\mathbf{R}^{N}} U_{\lambda_{n}, a_{n}}^{p-1} w_{n}^{2} d x\right\} \\
& +o\left(\frac{\lambda_{n}^{2}}{d_{n}^{2}}\right)+o\left(\varepsilon_{n} \lambda_{n}^{2}\left|\log \lambda_{n}\right|\right)+o\left(\left\|\nabla w_{n}\right\|_{L^{2}(\Omega)}^{2}\right) \\
& \geq S+C_{1}\left(\frac{\lambda_{n}^{2}}{d_{n}^{2}}\right)-C_{2} k\left(a_{n}\right) \varepsilon_{n} \lambda_{n}^{2}\left|\log \lambda_{n}\right| \\
& \geq S-\frac{\varepsilon_{n} k\left(a_{n}\right) C_{2}}{2 e} \exp \left(-\frac{2 C_{1}\left(\frac{1}{d_{n}^{2}}\right)}{\varepsilon_{n} k\left(a_{n}\right) C_{2}}\right),
\end{aligned}
$$

again we used (3.2).

On the other hand, Lemma 2.7 yields constants $C_{3}, C_{4}>0$ such that

$$
S_{\varepsilon_{n}, k} \leq S-C_{3} \varepsilon_{n} \exp \left(-\frac{C_{4}}{\varepsilon_{n}}\right) \text {. }
$$

Combining these, we obtain

$$
C_{5} k\left(a_{n}\right) \exp \left(-\frac{C_{6}\left(\frac{1}{d_{n}^{2}}\right)}{\varepsilon_{n} k\left(a_{n}\right)}\right) \geq 3 \exp \left(-\frac{C_{4}}{\varepsilon_{n}}\right)
$$

for some $C_{5}, C_{6}>0$. Taking logarithms of both sides and multiplying by $\varepsilon_{n}$, we have

$$
\varepsilon_{n} \log C_{5}+\varepsilon_{n} \log k\left(a_{n}\right)-\frac{C_{6}\left(\frac{1}{d_{n}^{2}}\right)}{k\left(a_{n}\right)} \geq \varepsilon_{n} \log C_{3}-C_{4} .
$$

Note that $\varepsilon_{n} \log k\left(a_{n}\right) \rightarrow 0$ as $n \rightarrow \infty$. Then the above inequality leads to the contradiction if $d_{n} \rightarrow 0$. Thus, $d_{n} \nrightarrow 0$.

Now, as before, we may drop $d_{n}$ in the expansion of $S_{\varepsilon_{n}, k}$ and

$$
\begin{aligned}
S_{\varepsilon_{n}, k} & \geq S+K_{3} R\left(a_{n}\right) \lambda_{n}^{2}-\varepsilon_{n} k\left(a_{n}\right) K_{4} \lambda_{n}^{2}\left|\log \lambda_{n}\right| \\
& +o\left(\lambda_{n}^{2}\right)+o\left(\varepsilon_{n} \lambda_{n}^{2}\left|\log \lambda_{n}\right|\right) \\
& \geq S+\left(K_{3} R\left(a_{n}\right)-p_{n}\right) \lambda_{n}^{2}-\varepsilon_{n}\left(K_{4} k\left(a_{n}\right)+q_{n}\right) \lambda_{n}^{2}\left|\log \lambda_{n}\right| \\
& \geq S-\left(\frac{K_{4} k\left(a_{n}\right)+q_{n}}{2 e}\right) \varepsilon_{n} \exp \left(-\frac{2\left(K_{3} R\left(a_{n}\right)-p_{n}\right)}{\left(K_{4} k\left(a_{n}\right)+q_{n}\right) \varepsilon_{n}}\right)
\end{aligned}
$$


where $p_{n}, q_{n}>0, p_{n}, q_{n}=o(1)$ and

$$
K_{3}=\frac{S}{2}\left(\frac{\omega_{4}^{2}}{A}\right), \quad K_{4}=\frac{S \omega_{4}}{8 A} .
$$

The last inequality of (3.4) follows again from (3.2).

Combining (3.4) with the upper bound Lemma 2.7 as before, we have

$$
\begin{aligned}
& S-\left(\frac{K_{4} k\left(a_{n}\right)+q_{n}}{2 e}\right) \varepsilon_{n} \exp \left(-\frac{2\left(K_{3} R\left(a_{n}\right)-p_{n}\right)}{\left(K_{4} k\left(a_{n}\right)+q_{n}\right) \varepsilon_{n}}\right) \\
& \leq S_{\varepsilon_{n}, k} \leq \\
& S-\left(\frac{K_{4} k(a)}{2 e}\right) \varepsilon_{n} \exp \left(-\frac{8 \omega_{4} R(a)+\varepsilon_{n} / e+2 \rho}{\varepsilon_{n} k(a)}\right)
\end{aligned}
$$

for any $a \in \Omega_{+}$and $\rho>0$. This leads to

$\varepsilon_{n} \log \left(K_{4} k\left(a_{n}\right)+q_{n}\right)-\frac{2\left(K_{3} R\left(a_{n}\right)-p_{n}\right)}{\left(K_{4} k\left(a_{n}\right)+q_{n}\right)} \geq \varepsilon_{n} \log \left(K_{4} k(a)\right)-\left(8 \omega_{4} R(a)+\varepsilon_{n} / e+2 \rho\right) / k(a)$.

Finally, letting $n \rightarrow \infty$ and then $\rho \rightarrow 0$, we obtain

$$
-8 \omega_{4} \frac{R\left(a_{\infty}\right)}{k\left(a_{\infty}\right)} \geq-8 \omega_{4} \frac{R(a)}{k(a)} .
$$

This completes the proof of Theorem.

Acknowledgements. Preparation of this work was partially supported by JSPS Grantin-Aid for Scientific Research, No. 17540186.

\section{References}

[1] H. Brezis, Elliptic equations with limiting Sobolev exponents. The impact of topology, Comm. Pure and Appl. Math. 39 (1986), 17-39.

[2] H. Brezis and L. Nirenberg, Positive solutions of nonlinear elliptic equations involving critical Sobolev exponents, Comm. Pure and Appl. Math. 36 (1983), 437-477.

[3] P. L. Lions, The concentration-compactness principle in the calculus of variations. The limit case. Part 1, Rev. Mat. Iberoamericana 1 (1985), 145-201.

[4] Z. C. Han, Asymptotic approach to singular solutions for nonlinear elliptic equations involving critical Sobolev exponent, Ann. Inst. Henri Poincaré 8 (1991), 159-174.

[5] T. Isobe, On the asymptotic analysis of H-systems, I: asymptotic behavior of large solutions, Adv. Diff. Eq. 6 (2001), 513-546.

[6] R. Molle and A. Pistoia, Concentration phenomena in elliptic problem with critical and supercritical growth, Adv. Diff. Eq. 8 (2003), 547-570.

[7] O. Rey, Proof of two conjectures of H. Brezis and L. A. Peletier, Manuscripta Math. 65 (1989), 19-37.

[8] O. Rey, The role of the Green's function in a non-linear elliptic equation involving the critical Sobolev exponent, J. Funct. Anal. 89 (1990), 1-52.

[9] F. Takahashi, On location of blow up points of least energy solutions to the Brezis-Nirenberg equations, Funkcialaj Ekvacioj 47 (2004), 145-166. 